OPEN

SUBJECT AREAS:

CLINICAL

PHARMACOLOGY

DRUG SAFETY

Received

12 November 2013

Accepted

25 February 2014

Published

12 March 2014

Correspondence and requests for materials should be addressed to H.J.C. (hichae@ chonbuk.ac.kr)

\title{
Soybean greatly reduces valproic acid plasma concentrations: A food-drug interaction study
}

\author{
Anu Marahatta' ${ }^{1}$ Bidur Bhandary' ${ }^{1}$ Seul-Ki Jeong ${ }^{2}$, Hyung-Ryong Kim ${ }^{3}$ \& Han-Jung Chae ${ }^{1}$
}

\begin{abstract}
'Department of Pharmacology, Medical School, Chonbuk National University, Jeonju, Korea 560-182, ${ }^{2}$ Department of Neurology, Medical School, Chonbuk National University, Jeonju, Korea, 561-182, ${ }^{3}$ Department of Dental Pharmacology, College of Dentistry, Wonkwang University, Iksan, Korea, 570-749.
\end{abstract}

The aim of this study was to investigate the effects of soy on the pharmacokinetics and pharmacodynamics of valproic acid (VPA). In a preclinical study, rats were pretreated with two different amounts of soy extract for five days $(150 \mathrm{mg} / \mathrm{kg}$ and $500 \mathrm{mg} / \mathrm{kg})$, which resulted in decreases of $57 \%$ and $65 \%$ in the $C_{\max }$ of VPA, respectively. AUC of VPA decreased to $83 \%$ and $70 \%$ in the soy pretreatment groups. Interestingly, the excretion rate of VPA glucuronide (VPAG) was higher in the soy-fed groups. Levels of UDP-glucuronosyltransferase (UGT) UGT1A3, UGT1A6, UGT2B7 and UGT2B15 were elevated in the soy-treated group, and GABA concentrations were elevated in the brain after VPA administration. However, this was less pronounced in soy extract pretreated group than for the untreated group. This is the first study to report the effects of soy pretreatment on the pharmacokinetics and pharmacodynamics of VPA in rodents.

$\mathrm{V}$ alproic acid (2-propylvaleric acid) (VPA) is an antiepileptic drug that enhances aminobutyric acid transmission $^{1}$ in the central nervous system. Valproic acid is also used as an alternative monotherapy to lithium for the treatment of acute mania, cyclothymia, mixed mania ${ }^{2}$ and anticancer agent ${ }^{3}$. VPA is tightly bound to proteins as a result of low clearance ${ }^{4}$, and VPA metabolism occurs via three routes: glucuronidation, $\beta$ oxidation, or cytochrome P450-mediated oxidation, the latter of which has a minor contribution to metabolism 5 . Valproate glucuronide is the major urinary metabolite of valproic acid and is metabolized by UGT1A3, UGT1A4, UGT1A6, UGT1A8, UGT1A9, UGT1A10, UGT2B7, and UGT2B15 6-9.

The global consumption of soybean preparations (soybean chips, soybean paste, soy sauce) has increased steadily over the last decade. Soy isoflavones may prevent cardiovascular disease $\mathrm{e}^{10}$ and postmenopausal osteoporosis, as well as increase lumbar spine bone mineral density to improve bone strength ${ }^{11}$. Soy isoflavones protect against liver damage by decreasing lipid peroxidation in a model of nonalcoholic steatohepatitis (NASH) induced by a methionine choline-deficient (MCD) diet ${ }^{12}$. Soy foods may also contribute to endometrial health ${ }^{13,14}$ and protect against prostate, breast, and colon cancer ${ }^{15,16}$. Whole soy foods contain a number of bioactive isoflavone components, consisting primarily of genistein and daidzein. Soy isoflavones have also been shown to reduce the efficacy of tamoxifen ${ }^{17}$. Genistein is known to inhibit mouse liver microsome CYP isozymes CYP1A and CYP2E dependent substrate metabolism in vitro ${ }^{18}$. Further, soy increases Phase II metabolism to promote faster detoxification and clearance of potentially carcinogenic intermediates. Soy products can also interfere with levothyroxine replacement ${ }^{19}$, warfarin ${ }^{20}$, and tamoxifen ${ }^{21}$. In view of these interactions, drugs with narrow therapeutic margins such as valproic acid should be carefully considered with respect to soy.

Metabolism and elimination of valproic acid are affected by glucuronidation, predominantly by uridine $5^{\prime}$ diphosphate-glucuronosyltransferases. Similarly, various studies have shown that soybeans induce UGT enzymes, essential components of glucuronidation ${ }^{22,23}$. Therefore, in this study, we investigated the effects of soybean on the pharmacokinetics and pharmacodynamics of VPA in rats.

\section{Results}

Concentration of VPA in plasma. To identify possible interactions between soy and VPA, we performed an analysis of plasma concentrations of VPA using rats pretreated with different amounts of soy extract $(150 \mathrm{mg} / \mathrm{kg}$ and $500 \mathrm{mg} / \mathrm{kg}$ ). Pretreatment with soy resulted in markedly lower plasma VPA concentrations compared with water alone (Figure 1). Specifically, VPA $C_{\max }$ values were decreased to $57 \%$ for $150 \mathrm{mg}$ soy and $65 \%$ for $500 \mathrm{mg}$ 


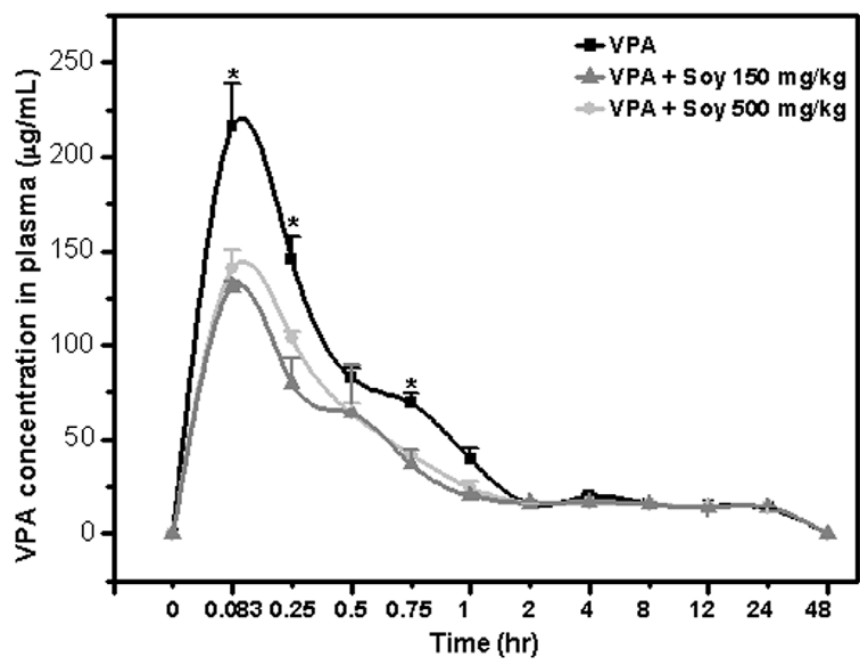

Figure 1 Soy inhibits the plasma concentration of valproic acid. Five days after rats were orally administered extracts of soy at 150 and $500 \mathrm{mg} /$ $\mathrm{kg}, 50 \mathrm{mg} / \mathrm{kg}$, VPA was injected IV, and blood was isolated at the indicated time points. Mean VPA concentration-time profiles following single iv injection $50 \mathrm{mg} / \mathrm{kg}$ with VPA only, VPA + Soy $150 \mathrm{mg}$ and VPA + Soy $500 \mathrm{mg}$ (error bars show SE, $\mathrm{n}=9$ ). VPA ( $\mathbf{\square}), \mathrm{VPA}+$ Soy $150 \mathrm{mg} / \mathrm{kg}(\boldsymbol{\Delta})$, $\mathrm{VPA}+$ Soy $500 \mathrm{mg} / \mathrm{kg}(\bigcirc)$.

soy treatments. The VPA $t_{\max }$ and MRT were not different among the treatments. Similarly, AUC decreased to $83 \%$ and $69 \%$ for $150 \mathrm{mg} / \mathrm{kg}$ and $500 \mathrm{mg} / \mathrm{kg}$ soy treatments, respectively. Clearance increased by $5 \%$ to $34 \%$ and the volume of distribution increased $70 \%$ in the soy treatment groups. There was a significant difference in $\mathrm{C}_{\max }, \mathrm{t}_{1 / 2}$, AUC, clearance, and distribution volume between the soy treatment and control groups (Table I).

Urinary VPAG and VPA excretion. VPA is metabolized to a glucuronide for easy removal through the urine ${ }^{24}$. Therefore, we measured the amounts of VPA and VPAG excreted in urine. Data on the excretion of VPAG in urine after the administration of VPA with and without soy is presented in Table II. VPAG concentration was 1.67 -fold and 1.44-fold higher in the $500 \mathrm{mg} / \mathrm{kg}$ and $150 \mathrm{mg} / \mathrm{kg}$ soy groups, respectively. Figure $2 \mathrm{~A}$ shows that the concentration of VPA in urine was more significantly decreased in the soy-pretreated groups compared with the group treated with VPA alone. Consistently, the level of VPAG excreted through urine was higher in the groups co-treated with soy (Figure 2B), suggesting that soy contributes to the highly effective excretion of VPA by enhancing the glucuronidation of VPA.

UGT mRNA enzyme induction. Soy treatment was previously reported to induce UGT enzyme expression in the liver ${ }^{22,23}$. To understand the mechanism of VPA and soy interaction, UGT enzyme gene expression was analyzed. Pretreatment with soy resulted in significantly higher UGT1A3, UGT1A6, UGT2B7, and UGT2B15 levels in the liver tissue compared to untreated rats. In the case of UGT2B15, the mRNA was increased to $150-200 \%$ in the soy pretreatment group compared with the control (Figure 3A). A fivefold increase was observed for UGT2B7 when pretreated with $500 \mathrm{mg}$ soy as compared to the control group (Figure $3 \mathrm{~B}$ ). Interestingly, for the soy-pretreated groups, a three- to four-fold mRNA increase was observed for UGT1A3 (Figure 3), while a two-fold increase was observed for UGT1A6 (Figure 3D). The data also showed that a higher dose of soy $(500 \mathrm{mg} / \mathrm{kg})$ more significantly induced UGT than the relatively lower dose of soy $(150 \mathrm{mg} / \mathrm{kg})$, which correlated with the pharmacokinetic profile of VPA (Figure 1).

UGT enzyme activity. Enzymatic activities were determined using selective substrates, CDCA, 4-MUB, AZT and BPA for UGT1A3 ${ }^{25}$, $\mathrm{UGT} 1 \mathrm{~A} 6^{26,27}$, UGT2B7 ${ }^{28}$ and UGT2B15 ${ }^{29}$ respectively. As shown in Figure 4, the hepatic microsomal enzymatic activities of four UGTs were found increase in case of soy treated group, compared to only VPA. UGT glucuronidation activity was found 2.0 (CDCA-glucuronide), 2.5 (4-MUB glucuronide), 1.8 (AZT glucuronide) and 1.6 (BPA glucuronide) fold increase in soy $500 \mathrm{mg}$ pretreated group compared to VPA only. Results of enzyme activity assay were generally consistent with that of mRNA determinations (Figure 3).

VPA and GABA concentrations in the brain. Since GABA is a neurotransmitter that explains the pharmacological effects of $\mathrm{VPA}^{30}$, GABA was measured in this study. GABA concentrations in the brain were increased within 5 min after VPA injection, and remained significantly elevated for $12 \mathrm{~h}$ thereafter (Figure $5 \mathrm{~A}$ ). In the soy-treated group, the concentrations of GABA in the brain were significantly inhibited throughout the entire study period; however, plasma levels of GABA were not different between the treatment and control groups (Supplementary Figure 1). To understand the increased concentrations of GABA in the brain, we measured concentrations of VPA. The concentrations of VPA in the brain decreased rapidly, whereas those of rats pre-treated with soy decreased more significantly compared with the group receiving a single treatment of VPA (Figure 5B). Although VPA was still measureable in the plasma $24 \mathrm{~h}$ after the $50 \mathrm{mg} / \mathrm{kg}$ dose, the levels in the brain fell below the limit of detection by that time. In addition, VPAG metabolites were not detectable in the brain (data not shown).

\section{Discussion}

When patients are prescribed a drug with a narrow therapeutic margin or are suffering from a specific pathological condition such as liver malfunction, food drug interactions may be an important issue for the patient's health. Enzyme inhibition or induction is important for drugs requiring therapeutic drug monitoring (TDM), and the seriousness of food-drug interactions depends on the therapeutic index of each drug. Drug-metabolizing enzymes convert lipophilic drugs into more hydrophilic compounds to facilitate elimination. Our results demonstrate that soy pretreatment for five days significantly altered the plasma pharmacokinetics of VPA and its metabolite VPAG. It has been reported that VPA is metabolized to a large extent by UGT enzymes, and we suggest that the decreased plasma

Table I | Pharmacokinetic data of valproic acid

\begin{tabular}{lccc} 
PK Parameter & VPA & VPA + Soy 150 mg & VPA + Soy 500 mg \\
\hline$C_{\text {max }}(\mu \mathrm{g} / \mathrm{mL})$ & $216.94 \pm 22.769$ & $124.636 \pm 6.861^{*}$ & $143.642 \pm 8.14 *$ \\
$t_{\max }(\mathrm{hr})$ & $0.083 \pm 0$ & $0.083 \pm 0$ & $0.083 \pm 0$ \\
$t_{1 / 2}(\mathrm{~h})$ & $3.953 \pm 0.121$ & $5.976 \pm 0.606^{*}$ & $4.980 \pm 0.154^{*}$ \\
AUC $_{0-24 \mathrm{hr}}$ & $490.287 \pm 12.851$ & $418.690 \pm 8.622^{*}$ & $335.296 \pm 29.777^{*}$ \\
AUC $_{0-\infty}$ & $656.579 \pm 11.453$ & $545.978 \pm 38.565$ & $456.491 \pm 44.750^{*}$ \\
Volume $(\mathrm{mL} / \mathrm{kg})$ & $503.492 \pm 22.248$ & $794.659 \pm 71.289 *$ & $857.967 \pm 67.056^{*}$ \\
CL_obs $(\mathrm{mL} / \mathrm{hr} / \mathrm{kg})$ & $88.028 \pm 1.569$ & $92.549 \pm 1.841$ & $118.974 \pm 7.793^{*}$ \\
MRT $(\mathrm{hr})$ & $8.747 \pm 0.257$ & $10.041 \pm 0.166$ & $6.801 \pm 0.981$ \\
\hline
\end{tabular}




\begin{tabular}{|c|c|c|c|}
\hline \multirow[b]{2}{*}{ Time (h) } & \multicolumn{3}{|c|}{ VPAG concentration $(\mu \mathrm{g} / \mathrm{mL})$} \\
\hline & Soy $500 \mathrm{mg}+$ VPA & Soy $150 \mathrm{mg}+$ VPA & VPA \\
\hline $0-2$ & $172.14=$ & $147.92 \pm$ & 102.48 \\
\hline $2-4$ & 146.81 & 131. & \\
\hline $4-6$ & $109.33 \pm 22.03$ & $89.35 \pm 22.03$ & $65.22 \pm 8.91$ \\
\hline $6-\varepsilon$ & $93.86 \pm 20.57$ & $65.38 \pm 5.30$ & $51.15 \pm 8.31$ \\
\hline & \pm 12.56 & $38.77=$ & $31.05 \pm$ \\
\hline & .42 & $29.44 \pm 0.98$ & $20.77 \pm 3.1$ \\
\hline & $6.24 \pm 0.51$ & $5.41 \pm 0.21$ & $4.47 \pm 2.35$ \\
\hline
\end{tabular}

exposure and increased $C_{\max }$ of VPA were caused by induction of UGT metabolism in the liver. Furthermore, our present observations indicate that the urinary VPAG and GABA concentrations in brain tissues were significantly different between the control and soytreated groups. The elimination $t_{1 / 2}$ of VPA was significantly increased in the soy-pretreated group. Together, these results suggest that a food/drug interaction exists for soy and VPA.

This study provides the first direct in vivo evidence that soybean decreases the serum concentration of VPA through interactions. In this study, the pharmacokinetic profiles of single-treatment VPA were similar to the findings of Yoshioka et al. ${ }^{31}$, who showed that soy has a significant effect on the $\mathrm{C}_{\max }$ of VPA but not on $t_{\max }$ value (Figure 1). Analysis of urine excreted 0-24 h after VPA consumption provided a much clearer view of the effect of soy (Figure 2A). Soy had a marked effect on excretion and thus absorption of VPA, as there was a parallel reduction in the concentration of VPA concentration in plasma. VPA is excreted extensively as glucuronide conjugates, which account for $30-70 \%$ of the administered dose ${ }^{6}$. In addition, the urinary VPAG excretion concentration in our study was found to be higher in the soy treatment group (Figure 2B). Specifically, urinary VPAG formation was 1.44-1.68 folds greater in the soy treatment groups compared to the untreated group, suggesting that the glucuronidation pathway is involved in the mechanism of interaction of VPA and soy.

UGTs, the primary enzyme family responsible for glucuronidation, were highly activated in the soy pre-treated groups. VPA is extensively metabolized in the liver, which occurs primarily by glucuronidation with probable contributions of UGT1A6, UGT1A9, and UGT2B7. VPA is also metabolized by a minor CYP-dependent oxidation pathway, which includes multiple enzymes such as CYP2A6, CYP2C9 and CYP2C19 ${ }^{32}$, suggesting that VPA is extensively metabolized through hepatic enzyme pathways. Hitomi Mori showed that activation of UGT by the antibiotic carbapenem results in decreased VPA plasma concentrations ${ }^{24}$. Recently, it was reported that serum level of VPA is also decreased by combined treatment with carbapenem, panipenem and meropenem ${ }^{33,34}$. Co-administration with oral contraceptives decreases concentrations of VPA, possibly as a result of UGT induction by ethinyl estradiol. As described above, the induction of UGT is generally believed to be responsible for the mechanism of interactions with VPA. We have shown that UGT mRNA as well as activity, which is mainly responsible for the metabolism of VPA, was significantly induced by soy pretreatment for five days (Figure 3 and 4). Similarly, UGT was induced in rats fed soy for one and two weeks ${ }^{35}$. There are several other reports that have demonstrated that soy foods increase UGT activity ${ }^{36-38}$. The major soybean-specific flavonoids genistein, daidzein and glycitein account for up to $50 \%, 40 \%$ and $10 \%$ of the total abundance of soybean isoflavones, respectively ${ }^{39}$. Soy isoflavones and flavonoids increase hepatic UGT activity in rodents ${ }^{35,37}$. Similarly, genistein has been reported to induce UGT activity ${ }^{40,41}$. Although we did not test the effects of each component of soybean on UGT, the isoflavone


Figure $2 \mid$ Soy enhances the elimination of valproic acid through the formation of glucuronide of valproic acid. Five days after rats were orally administered extracts of soy at 150 and $500 \mathrm{mg} / \mathrm{kg}, 50 \mathrm{mg} / \mathrm{kg}$, VPA was injected IV, and urine was isolated at the indicated time points. Mean urinary VPA (A) and VPAG (B) concentration-time profiles following single iv injection $50 \mathrm{mg} / \mathrm{kg}$ with VPA only, VPA + Soy $150 \mathrm{mg} / \mathrm{kg}$ and $\mathrm{VPA}+$ Soy $500 \mathrm{mg} / \mathrm{kg}$ (error bars show SE, $\mathrm{n}=9$. VPA (O), VPA + Soy $150 \mathrm{mg} / \mathrm{kg}(\mathbf{\Delta}), \mathrm{VPA}+$ Soy $500 \mathrm{mg} / \mathrm{kg}(\boldsymbol{\square})$.

components do appear to contribute to drug and food interactions through the induction of the UGT enzymes.

In the present study, GABA was used as a pharmacodynamic marker for the concentration of VPA. We found that the brain GABA concentration after VPA treatment was lower in the soy pretreatment group compared to the control group (Figure 5A). Although the concentration of VPA decreased sharply within one hour (Figure 5B), the concentration of GABA was highly increased and maintained through the whole period up to12 hours. The cotreatment of soy greatly inhibited the concentrations of both GABA and VPA in brain. However, no significant difference in the serum GABA concentrations with or without soy was observed (Supplementary Figure 1). Similarly, VPA was found to increase GABA levels in the whole brain and several brain regions in rodents ${ }^{42-44}$. Similar to the kinetic profile of GABA concentrations observed in our study, GABA concentrations were previously shown to be increased within $5 \mathrm{~min}$ after VPA injection and to remain significantly elevated for at least $8 \mathrm{~h}$ thereafter ${ }^{45}$. Thus, the results of the present study indicate that soy affects the concentration of VPA, 
A

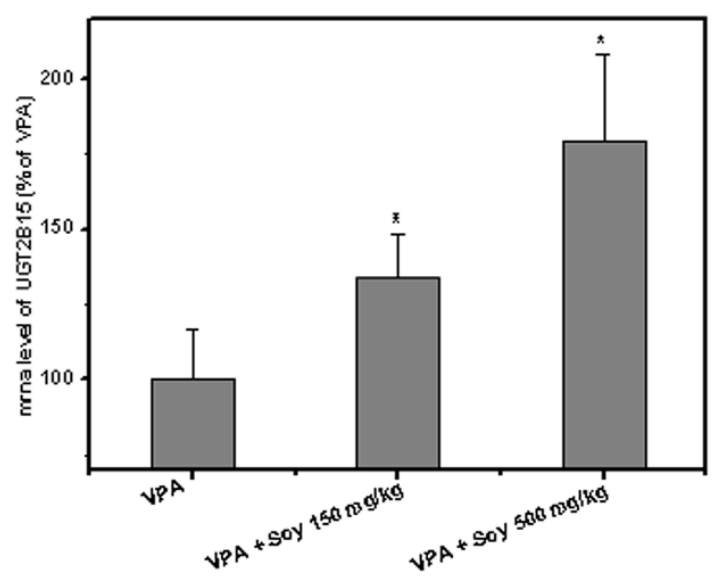

C

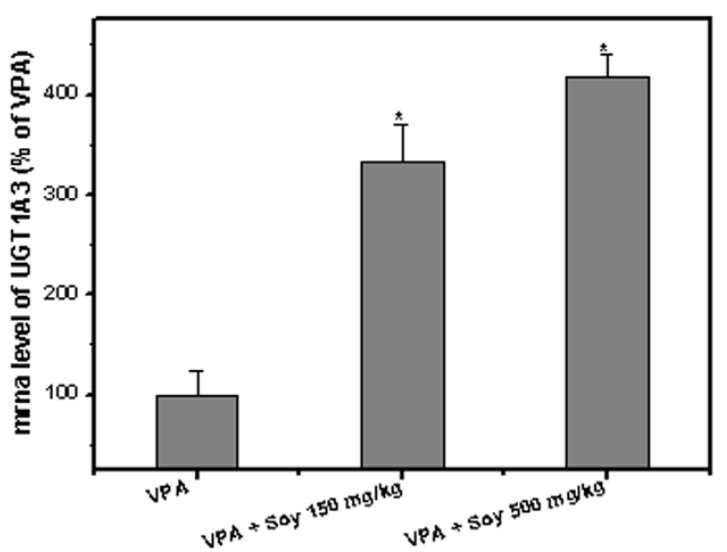

B

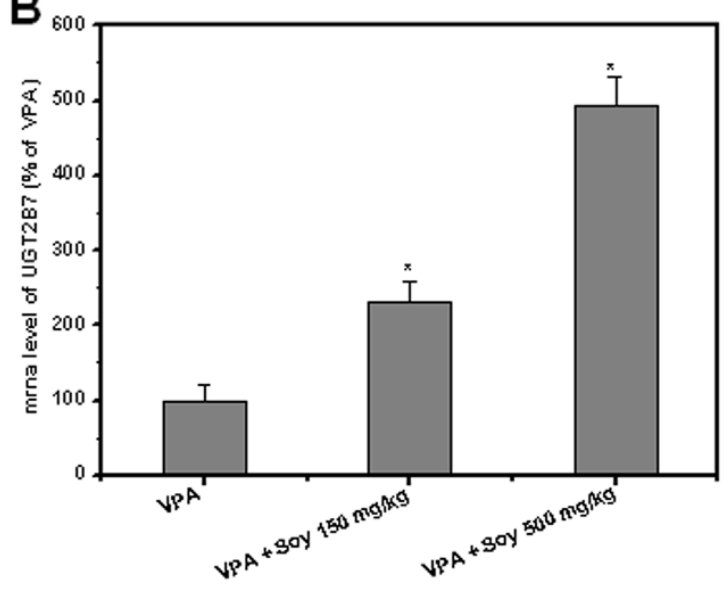

D

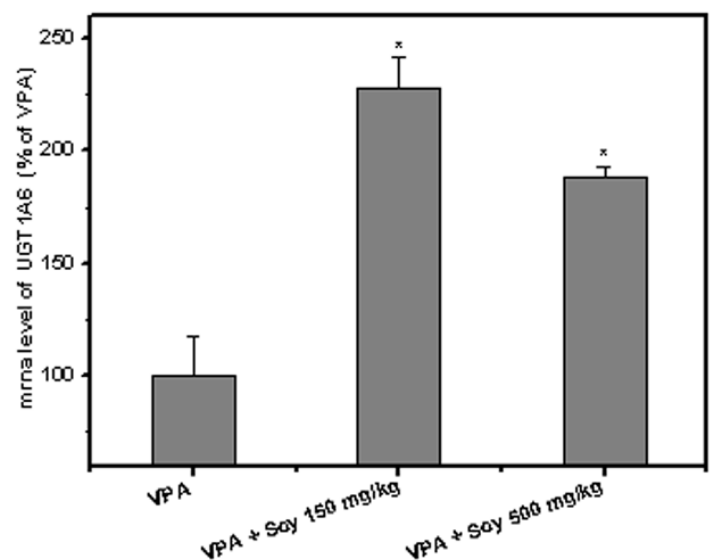

Figure 3 Induction of UGT enzymes in soy pretreated rats compared to untreated. Five days after rats were orally administered extracts of soy at 150 and $500 \mathrm{mg} / \mathrm{kg}, 50 \mathrm{mg} / \mathrm{kg}$, VPA was injected iv. UGT2B15 (A), UGT2B7 (B), UGT1A3(C) and UGT1A6 mRNA (D) were analyzed by real-time PCR assay.

resulting in a pharmacodynamic effect on GABA concentration in the brain.

In conclusion, the results of this study provide the first evidence for differences in pharmacokinetic and pharmacodynamic effects following pretreatment with soy. We identified soy extract as an inducer of liver UGT in rats. Together, our data suggest that pretreatment with soy induces the induction of UGT, resulting in changes in the pharmacokinetic and pharmacodynamic profiles of VPA. The ingestion of VPA with soy extract was also associated with a decrease in VPA bioavailability, and this pharmacokinetic interaction may lead to a clinically significant decrease in GABA concentration. This study suggests a new mechanism to account for soy-induced food-drug interactions. Further clinical study is warranted to explore the detailed implications of these findings.

\section{Methods}

Materials. Acetonitrile and formic acid were purchased from Fisher Scientific (Pittsburgh, PA, USA). Sodium valproate (VPA, $300 \mathrm{mg} / 3 \mathrm{~mL}$ ampoules) was provided by Chonbuk National Hospital. Valproic acid $\beta$-D glucuronide (VPAG) was purchased from Santa Cruz Biotechnology, Inc. (Santa Cruz, CA, USA). Chenodeoxycholic acid 24-acyl $\beta$-D-glucuronide, $3^{\prime}$-Azido- $3^{\prime}$-deoxythymidine $\beta$-Dglucuronide and bisophenol A $\beta$-D-glucuronide was purchased from Toronto research chemical (Ontario, Canada). Nonanoic acid, gamma aminobutyric acid, ammonium formate, Uridine $5^{\prime}$-diphosphoglucuronic acid trisodium salt (UDPGA), $\mathrm{MgCl}_{2}$, Saccharic acid 1,4 lactone, chenodeoxycholic acid (CDCA), Azidothymidine (AZT), Bisophenol A (BPA), 4-methylumbelliferone (4-MUB) and 4methylumbelliferyl- $\beta$-D- glucuronide hydrate were purchased from Sigma (St. Louis, MO, USA). Water was purified using a Milli-Q system from Millipore (Bedford, MA, USA).
Preparation of soy extract. Soybeans were purchased from a local Jeonju market (South Korea). First, the beans were ground and powdered, and the resulting soybean powder was defatted with petroleum ether and sonicated twice with $1800 \mathrm{~mL}$ of $95 \%$ ethanol for $3 \mathrm{~h}$ at $50^{\circ} \mathrm{C}$. Filtrates were concentrated using a vacuum evaporator at $50^{\circ} \mathrm{C}$ and powdered by freeze-drying.

Animal treatment. Male Sprague-Dawley rats aged 6-8 weeks were purchased from Samatako (Deajeon, South Korea). The rats were synchronized with a light/dark cycle to adjust circadian rhythms. Rats were fasted overnight prior to initiating experiments and were allowed free access to tap water. The rats were divided into three groups: rats in Group I were fed $500 \mathrm{mg} / \mathrm{kg}$ soy extract twice daily by gavage for five days, rats in Group II were fed $100 \mathrm{mg} / \mathrm{kg}$ soy extract twice daily by gavage for five days, and rats in the Group III control were fed an equal volume of water for five days. After the last soy extract treatment, access to the diet was removed and only water was provided. On the sixth day, rats were injected with $50 \mathrm{mg} / \mathrm{kg}$ VPA through the femoral vein.

Animal care. All animal procedures were performed according to the Guidelines for the Care and Use of Laboratory Animals approved by the Chonbuk National University Committee of Ethics of Animal Experimentation.

Blood and urine sampling. Blood samples were collected at the following time points: $0,0.083,0.25,0.5,0.75,1,2,4,8,12$, and $24 \mathrm{~h}$ after dosing. The plasma samples were separated by centrifugation at 2,000 $\times \mathrm{g}$ for $10 \mathrm{~min}$, and the plasma was transferred into eppendorf tubes and stored at $-80^{\circ} \mathrm{C}$ until analyzed. Urine samples were also collected every $2 \mathrm{~h}$ for $24 \mathrm{~h}$.

Quantification of VPA and VPAG in plasma and urine. Quantification of VPA and VPAG in serum and urine were determined by HPLC coupled with mass spectrometry. To a $100 \mu \mathrm{L}$ plasma/urine sample, $100 \mu \mathrm{L}$ of IS and $800 \mu \mathrm{L}$ of acetonitrile were added. The resulting solution was vortexed for $30 \mathrm{sec}$ and centrifuged at $13,000 \times \mathrm{g}$ for $20 \mathrm{~min}$. Next, the supernatant was transferred to an HPLC vial for injection. HPLC separation was performed on an Agilent 1100 system (Agilent, Palo Alto, CA, USA). Chromatography separation was performed using a 



C



c

Figure $4 \mid$ Measurement of glucuronidation enzyme activity. (A) CDCA-24 glucuronide (UGT1A3), (B) 4-MUB glucuronide (UGT1A6), (C) AZT glucuronide (UGT2B7) and (D) BPA glucuronide (UGT2B15) in liver microsomes from soy pretreated and untreated rats. VPA iv was injected five days after oral administration of soy extract (150 and $500 \mathrm{mg} / \mathrm{kg}$ ).

Zorbax Eclipse XDB-C18 $(150 \times 2.1 \mathrm{~mm}$ internal diameter, $3.5 \mu \mathrm{m}$ particle size $)$. The mobile phase was composed of solvent $\mathrm{A}, 10 \mathrm{mM}$ ammonium formate, and solvent $\mathrm{B}$, acetonitrile. VPA, VPAG, and IS quantifications were achieved using negative ion-mode electrospray ionization with multiple reaction monitoring (MRM). The MRM transitions of $\mathrm{m} / \mathrm{z} 319$ to 174.8 for VPAG, $\mathrm{m} / \mathrm{z} 143$ to 143 , and $\mathrm{m} / \mathrm{z}$ 157 to 157 were used for analysis.

Quantification of GABA. Brains were removed, weighed, dissected, and homogenized in trichloroacetic acid. Acetaminophen was used as an internal standard. Zorbax columns were used for quantification of GABA. The mobile phase A consisted of $0.1 \%$ formic acid in water, and the mobile phase B consisted of $0.1 \%$ formic acid in acetonitrile. The specific ions $(\mathrm{m} / \mathrm{z})$ monitored were GABA $(\mathrm{m} / \mathrm{z} 104$ to 87) and acetaminophen (m/z 151.7 to 109.7$)$.

Total RNA isolation and real-time PCR. Six hours after the IV injection of VPA, rats were scarified, and liver samples were extracted. Approximately $100 \mathrm{mg}$ frozen liver tissue was pulverized in liquid nitrogen, and total RNA was extracted using Trizol reagent according to the manufacturer's instruction. RNA was quantified and purity was monitored by spectrometry (Eppendorf Bio Photometer). The expression levels ofUGT1A6, UGT1A3, UGT2B7 and UGT2B15 in rat liver tissues were measured by quantitative real-time PCR on an ABI PRISM (Applied Biosystems, NJ, USA). PCR amplification was performed as described previously. The primer pair sequences are shown in Supplementary Table I.

Microsome preparation. Hepatic microsomes were prepared as described previously by Lee et $\mathrm{al}^{46}$. Liver samples were resuspended in buffer A $(250 \mathrm{mM}$ sucrose, $20 \mathrm{mM}$ HEPES, pH 7.5, $10 \mathrm{mM} \mathrm{KCL}, 1.5 \mathrm{mM} \mathrm{MgCl} 2,1 \mathrm{mM}$ EDTA, 1 mM EGTA and $1 \times$ protease inhibitor complex for $30 \mathrm{~min}$. The lysates were then homogenized and centrifused at $750 \times \mathrm{g}$ for $10 \mathrm{~min}$ at $4^{\circ} \mathrm{C}$. The supernant from the homogenized lysates was then centrifused at $100,000 \times \mathrm{g}$ for $1 \mathrm{~h}$ at $4^{\circ} \mathrm{C}$. Liver microsomes were resuspended in $50 \mathrm{mM}$ Tris- $\mathrm{HCl}, \mathrm{pH} 7.4$, containing $0.25 \mathrm{M}$ sucrose and $1 \mathrm{mM}$ dithiothreitol.

Enzyme glucuronidation activity 4-MUB glucuronidation activity (UG1 A6). UGT activity with 4-MUB as substrate was measured in the microsomes as described previously ${ }^{47}$ by using Molecular device spectra MAX GEMINI EM spectrofluorophotometer. $100 \mu \mathrm{g}$ microsomal proteins were used.

CDCA-24 glucuronidation (UGT1A3) and AZT glucuronidation (UGT2B7). The incubation mixtures were prepared in a total volume $100 \mu \mathrm{L}$, as follows: rat liver microsomes $(0.1 \mathrm{mg}), 10 \mathrm{mM} \mathrm{MgCl}_{2}, 4 \mathrm{mM}$ UDPGA, UGT specific probe substrate $67.5 \mu \mathrm{M}$ CDCA for UGT1A3 and $100 \mu \mathrm{M}$ AZT for UGT 2B7. Reaction were initiated by addition of UDPGA and incubations were carried out at $37^{\circ} \mathrm{C}$ in a shaking water bath for $60 \mathrm{~min}$. Reaction was terminated by addition of ice cold methanol $(100 \mu \mathrm{L})$. The incubation mixtures were centrifuged at $13,000 \times \mathrm{g}$ for $10 \mathrm{~min}$. Aliquots $(3 \mu \mathrm{L})$ were then injected onto the LC/MS/MS system. Glucuronides produced from UGT specific substrates were respectively determined by LC/MS/MS. Separation was performed on Kinetex C18 $(50 \times 2.10 \mathrm{~mm}$ internal diameter and $2.6 \mu \mathrm{m}$ particle size). The mobile phase was composed of solvent $\mathrm{A}$, water and solvent $\mathrm{B}$, acetonitrile, and both solvent containing $0.1 \%$ formic acid. The gradient run started with $10 \%$ solvent B for $1 \mathrm{~min}$ and then raised to $100 \%$ for $6 \mathrm{~min}$, where it remained for $1 \mathrm{~min}$ and then returned to $10 \% \mathrm{~B}$ in $10 \mathrm{~min}$ and, then maintained for $5 \mathrm{~min}$. Flow rate was $0.3 \mathrm{~mL} / \mathrm{min}$ and run time was $15 \mathrm{~min}$.

BPA glucuronidation (UGT2B15). The incubation mixture contained BPA $100 \mu \mathrm{M}$ as a substrate, microsome $(0.2 \mathrm{mg}), 10 \mathrm{mM} \mathrm{MgCl} 2,5 \mathrm{mM}$ UDPGA in a final volume of $200 \mu \mathrm{L}$. Incubation was performed for $30 \mathrm{~min}$ at $37^{\circ} \mathrm{C}$ and terminated by adding adding $100 \mu \mathrm{L}$ of $15 \%$ perchloric acid and vertexing. The samples were centrifuged at $13,000 \times \mathrm{g}$ for $15 \mathrm{~min}$ and the supernantant was injected in LC-MS/MS analysis. Chromatography separation was performed using a Zorbax Eclipse XDB-C18 $(150 \times$ $2.1 \mathrm{~mm}$ internal diameter, $3.5 \mu \mathrm{m}$ particle size). Gradient elution with water (solvent 
A



B

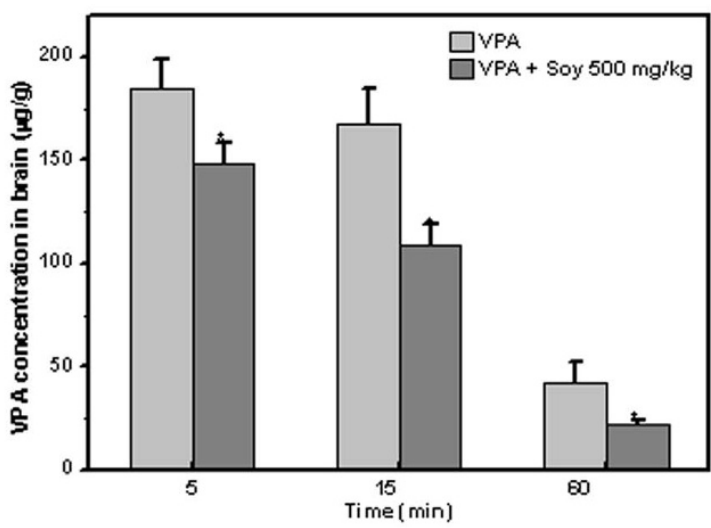

Figure 5 Soy inhibits the concentrations of brain GABA (A) and VPA (B) in valproic acid-treated rats. Five days after rats were orally administered extracts of soy at 150 and $500 \mathrm{mg} / \mathrm{kg}, 50 \mathrm{mg} / \mathrm{kg}$, VPA was injected IV, and brains were isolated at the indicated time points.

A) and acetonitrile (solvent B) with the following conditions was applied:0\% B for $1 \mathrm{~min}$, followed by a linear gradient to $90 \% \mathrm{~B}$ within $5 \mathrm{~min}$ and then return to $0 \% \mathrm{~B}$ in $10 \mathrm{~min}$ and maintained for $5 \mathrm{~min}$. Flow rate was $0.4 \mathrm{~mL} / \mathrm{min}$ and run time was $15 \mathrm{~min}$.

An Agilent Technologies 6410 triple quadrupole mass spectrometer equipped with electrospray ionization was used. The following conditions were used for analysis: capillary voltage $4 \mathrm{kV}$, gas temperature $300^{\circ} \mathrm{C}$ and gas flow $10 \mathrm{~L} / \mathrm{min}$. Samples were analyzed by MRM mode in negative ionization mode. The MRM transitions of $\mathrm{m} / \mathrm{z}$ 567.4 to 391.3 for CDCA-G, m/z 442.1 to 125 for AZT-G, and m/z 403 to 227 for BPA$\mathrm{G}$ were used for analysis.

Pharmacokinetic analysis. The pharmacokinetic profiles of VPA were obtained by non-compartmental analysis using WinNonlin version 5.2.1 (Pharsight, Sunnyvale, CA, USA). The area under the plasma concentration-time curve (AUC) was calculated using the linear trapezoidal method. The maximum plasma concentration $\left(\mathrm{C}_{\max }\right)$ and the time to reach the maximum plasma concentration $\left(\mathrm{t}_{\max }\right)$ were calculated from the experimental data. The elimination rate constant (Kel) was estimated by regression analysis from the slope the line of best fit, and the half-life $\left(t_{1 / 2}\right)$ of the drug was obtained as $0.693 / \mathrm{Kel}$. The dose/AUC values were used to calculate total plasma clearance.

Statistical considerations. Pharmacokinetic data are presented as mean $\pm \mathrm{SD}$. The statistical significance of results was analyzed using one-way ANOVA. Statistical significance was performed at $\mathrm{P}<0.05$. ${ }^{*}$ Difference between VPA only and VPA plus Soy treatment, $\mathrm{P}<0.05$.

1. Czuczwar, S. J. \& Patsalos, P. N. The new generation of GABA enhancers. Potential in the treatment of epilepsy. CNS Drugs 15, 339-50 (2001).

2. Lennkh, C. \& Simhandl, C. Current aspects of valproate in bipolar disorder. Int Clin Psychopharmacol 15, 1-11 (2000).

3. Slingerland, M., Guchelaar, H. J. \& Gelderblom, H. Histone deacetylase inhibitors: an overview of the clinical studies in solid tumors. Anticancer Drugs 25, 140-9 (2014)
4. Cloyd, J. C. \& Remmel, R. P. Antiepileptic drug pharmacokinetics and interactions: impact on treatment of epilepsy. Pharmacotherapy 20, 139S-151S (2000).

5. Kumar, S. et al. Disposition of valproic acid in maternal, fetal, and newborn sheep. II: metabolism and renal elimination. Drug Metab Dispos 28, 857-64 (2000).

6. Argikar, U. A. \& Remmel, R. P. Effect of aging on glucuronidation of valproic acid in human liver microsomes and the role of UDP-glucuronosyltransferase UGT1A4, UGT1A8, and UGT1A10. Drug Metab Dispos 37, 229-36 (2009).

7. Krishnaswamy, S. et al. UDP glucuronosyltransferase (UGT) 1 A6 pharmacogenetics: II. Functional impact of the three most common nonsynonymous UGT1A6 polymorphisms (S7A, T181A, and R184S). J Pharmacol Exp Ther 313, 1340-6 (2005).

8. Chung, J. Y. et al. Pharmacokinetic and pharmacodynamic interaction of lorazepam and valproic acid in relation to UGT2B7 genetic polymorphism in healthy subjects. Clin Pharmacol Ther 83, 595-600 (2008).

9. Ethell, B. T., Anderson, G. D. \& Burchell, B. The effect of valproic acid on drug and steroid glucuronidation by expressed human UDP-glucuronosyltransferases. Biochem Pharmacol 65, 1441-9 (2003).

10. Wenzel, U., Fuchs, D. \& Daniel, H. Protective effects of soy-isoflavones in cardiovascular disease. Identification of molecular targets. Hamostaseologie 28, 85-8 (2008).

11. Taku, K. et al. Effect of soy isoflavone extract supplements on bone mineral density in menopausal women: meta-analysis of randomized controlled trials Asia Pac J Clin Nutr 19, 33-42 (2010).

12. Ustundag, B. et al. Protective effect of soy isoflavones and activity levels of plasma paraoxonase and arylesterase in the experimental nonalcoholic steatohepatitis model. Dig Dis Sci 52, 2006-14 (2007).

13. $\mathrm{Xu}, \mathrm{W}$. H. et al. Interaction of soy food and tea consumption with CYP19A1 genetic polymorphisms in the development of endometrial cancer. Am J Epidemiol 166, 1420-30 (2007).

14. $\mathrm{Xu}, \mathrm{W}$. H. et al. Soya food intake and risk of endometrial cancer among Chinese women in Shanghai: population based case-control study. BMJ 328, 1285 (2004).

15. Badger, T. M., Ronis, M. J., Simmen, R. C. \& Simmen, F. A. Soy protein isolate and protection against cancer. J Am Coll Nutr 24, 146S-149S (2005).

16. Douglas, C. C., Johnson, S. A. \& Arjmandi, B. H. Soy and its isoflavones: the truth behind the science in breast cancer. Anticancer Agents Med Chem 13, 1178-87 (2013).

17. Jones, J. L., Daley, B. J., Enderson, B. L., Zhou, J. R. \& Karlstad, M. D. Genistein inhibits tamoxifen effects on cell proliferation and cell cycle arrest in T47D breast cancer cells. Am Surg 68, 575-7; discussion 577-8 (2002).

18. Helsby, N. A., Chipman, J. K., Gescher, A. \& Kerr, D. Inhibition of mouse and human CYP 1A- and 2E1-dependent substrate metabolism by the isoflavonoids genistein and equol. Food Chem Toxicol 36, 375-82 (1998).

19. Fruzza, A. G., Demeterco-Berggren, C. \& Jones, K. L. Unawareness of the effects of soy intake on the management of congenital hypothyroidism. Pediatrics 130, e699-702 (2012).

20. Nutescu, E. A., Shapiro, N. L., Ibrahim, S. \& West, P. Warfarin and its interactions with foods, herbs and other dietary supplements. Expert Opin Drug Saf 5, 433-51 (2006).

21. Ju, Y. H., Doerge, D. R., Allred, K. F., Allred, C. D. \& Helferich, W. G. Dietary genistein negates the inhibitory effect of tamoxifen on growth of estrogendependent human breast cancer (MCF-7) cells implanted in athymic mice. Cancer Res 62, 2474-7 (2002).

22. Appelt, L. C. \& Reicks, M. M. Soy induces phase II enzymes but does not inhibit dimethylbenz[a] anthracene-induced carcinogenesis in female rats. J Nutr 129, 1820-6 (1999).

23. Froyen, E. B., Reeves, J. L., Mitchell, A. E. \& Steinberg, F. M. Regulation of phase II enzymes by genistein and daidzein in male and female Swiss Webster mice. J Med Food 12, 1227-37 (2009).

24. Mori, H., Takahashi, K. \& Mizutani, T. Interaction between valproic acid and carbapenem antibiotics. Drug Metab Rev 39, 647-57 (2007).

25. Erichsen, T. J. et al. Regulation of the human bile acid UDPglucuronosyltransferase $1 \mathrm{~A} 3$ by the farnesoid X receptor and bile acids. J Hepatol 52, 570-8 (2010).

26. Hanioka, N., Jinno, H., Tanaka-Kagawa, T., Nishimura, T. \& Ando, M. Determination of UDP-glucuronosyltransferase UGT1A6 activity in human and rat liver microsomes by HPLC with UV detection. J Pharm Biomed Anal 25, 65-75 (2001).

27. Heurtaux, T. et al. Induction of UGT1A6 isoform by inflammatory conditions in rat astrocytes. Neuropharmacology 50, 317-28 (2006).

28. Court, M. H. et al. Evaluation of $3^{\prime}$-azido-3'-deoxythymidine, morphine, and codeine as probe substrates for UDP-glucuronosyltransferase 2B7 (UGT2B7) in human liver microsomes: specificity and influence of the UGT2B7*2 polymorphism. Drug Metab Dispos 31, 1125-33 (2003).

29. Hanioka, N., Oka, H., Nagaoka, K., Ikushiro, S. \& Narimatsu, S. Effect of UDPglucuronosyltransferase $2 \mathrm{~B} 15$ polymorphism on bisphenol A glucuronidation. Arch Toxicol 85, 1373-81 (2011).

30. Guidotti, A. et al. Epigenetic GABAergic targets in schizophrenia and bipolar disorder. Neuropharmacology 60, 1007-16 (2011).

31. Yoshioka, H. et al. Effects of lithium on the pharmacokinetics of valproate in rats. J Pharm Pharmacol 52, 297-301 (2000). 
32. Ragueneau-Majlessi, I. et al. Lack of pharmacokinetic interactions between steady-state zonisamide and valproic acid in patients with epilepsy. Clin Pharmacokinet 44, 517-23 (2005).

33. Nagai, K. et al. Decrease in serum levels of valproic acid during treatment with a new carbapenem, panipenem/betamipron. J Antimicrob Chemother 39, 295-6 (1997).

34. De Turck, B. J. et al. Lowering of plasma valproic acid concentrations during concomitant therapy with meropenem and amikacin. J Antimicrob Chemother $\mathbf{4 2}$ 563-4 (1998).

35. Appelt, L. C. \& Reicks, M. M. Soy feeding induces phase II enzymes in rat tissues. Nutr Cancer 28, 270-5 (1997).

36. Navarro, S. L. et al. UGT1A6 and UGT2B15 polymorphisms and acetaminophen conjugation in response to a randomized, controlled diet of select fruits and vegetables. Drug Metab Dispos 39, 1650-7 (2011).

37. Siess, M. H., Le Bon, A. M. \& Suschetet, M. Dietary modification of drugmetabolizing enzyme activities: dose-response effect of flavonoids. $J$ Toxicol Environ Health 35, 141-52 (1992).

38. Mirsalis, J. C., Hamilton, C. M., Schindler, J. E., Green, C. E. \& Dabbs, J. E. Effects of soya bean flakes and liquorice root extract on enzyme induction and toxicity in B6C3F1 mice. Food Chem Toxicol 31, 343-50 (1993).

39. Martinez-Montemayor, M. M. et al. Individual and combined soy isoflavones exert differential effects on metastatic cancer progression. Clin Exp Metastasis 27, 465-80 (2010)

40. Walle, T., Otake, Y., Galijatovic, A., Ritter, J. K. \& Walle, U. K. Induction of UDPglucuronosyltransferase UGT1A1 by the flavonoid chrysin in the human hepatoma cell line hep G2. Drug Metab Dispos 28, 1077-82 (2000).

41. Galijatovic, A., Otake, Y., Walle, U. K. \& Walle, T. Induction of UDP glucuronosyltransferase UGT1A1 by the flavonoid chrysin in Caco-2 cells-potential role in carcinogen bioinactivation. Pharm Res 18, 374-9 (2001).

42. Chapman, A., Keane, P. E., Meldrum, B. S., Simiand, J. \& Vernieres, J. C. Mechanism of anticonvulsant action of valproate. Prog Neurobiol 19, 315-59 (1982).

43. Johnston, D. Valproic acid: update on its mechanisms of action. Epilepsia 25 Suppl 1, S1-4 (1984)

44. Morre, M., Keane, P. E., Vernieres, J. C., Simiand, J. \& Roncucci, R. Valproate: recent findings and perspectives. Epilepsia 25 Suppl 1, S5-9 (1984).
45. Nau, H. \& Loscher, W. Valproic acid: brain and plasma levels of the drug and its metabolites, anticonvulsant effects and gamma-aminobutyric acid (GABA) metabolism in the mouse. J Pharmacol Exp Ther 220, 654-9 (1982).

46. Lee, G. H. et al. The roles of ER stress and P450 2E1 in $\mathrm{CCl}(4)$-induced steatosis. Int J Biochem Cell Biol 43, 1469-82 (2011).

47. van der Logt, E. M., Roelofs, H. M., Nagengast, F. M. \& Peters, W. H. Induction of rat hepatic and intestinal UDP-glucuronosyltransferases by naturally occurring dietary anticarcinogens. Carcinogenesis 24, 1651-6 (2003).

\section{Acknowledgments}

This study was supported by the National Research Foundation (2012R1A2A1A3001907 and 2008-0062279).

\section{Author contributions}

A.M. and H.J.C. designed the experiment. A.M. and B.B. conducted the experiments, and participated in the data analysis, interpretation and preparation of manuscript. S.K.J. and H.R.K. give suggestion while preparing manuscript. All the authors reviewed the manuscript.

\section{Additional information}

Supplementary information accompanies this paper at http://www.nature.com/ scientificreports

Competing financial interests: The authors declare no competing financial interests.

How to cite this article: Marahatta, A., Bhandary, B., Jeong, S.-K., Kim, H.-R. \& Chae, H.-J. Soybean greatly reduces valproic acid plasma concentrations: A food-drug interaction study. Sci. Rep. 4, 4362; DOI:10.1038/srep04362 (2014).

This work is licensed under a Creative Commons AttributionSA NonCommercial-ShareAlike 3.0 Unported license. To view a copy of this license, visit http://creativecommons.org/licenses/by-nc-sa/3.0 